\title{
Research Progress of Taijiquan Intervention in Rehabilitation of Patients with Spinal Cord Injury
}

\author{
Shibao Xu, Youqun Tan \\ School of Physical Education, Jiangxi Normal University, China
}

Keywords: Taijiquan; Tai Chi rehabilitation; Tai Chi in water.

\begin{abstract}
Through the methods of literature review and logical thinking, this paper discusses the research progress of Taijiquan in the rehabilitation treatment of patients with spinal cord injury, and provides theoretical basis for the rehabilitation treatment of patients with spinal cord injury.
\end{abstract}

\section{Introduction}

With the development of economic level in the world, the incidence of spinal cord injury is increasing year by year. According to the World Health Organization, the incidence of spinal cord injury is one-thousandth. There are at least 1.3 million people with spinal cord injury in China, and it is increasing at a rate of 50,000 to 70,000 per year. $90 \%$ of spinal cord injuries are caused by trauma. The high incidence age is 20 to 30 years old. Most of them are caused by traffic accidents, falls, violence and spinal cord diseases, which bring heavy burden to families and society. Spinal cord injury patients are often accompanied by varying degrees of dysfunction. Taijiquan exercise can improve the motor ability, sensory function, daily living ability of patients with spinal cord injury and reduce the incidence of complications; it is very helpful to the recovery of hemiplegia after central nervous system injury, and has a good preventive effect on the prevention of falls. . With the application of Taijiquan in the medical field, this article reviews the literature on rehabilitation treatment of spinal cord injury patients with Taijiquan.

\section{Application of Shadowboxing In Rehabilitation Of Patients With Spinal Cord Injury}

In 2006, Li Pinmei et al. first put the Taijiquan training into the rehabilitation treatment of patients with spinal cord injury, and through clinical practice proved that Taijiquan rehabilitation is an effective method to treat hemiplegia, can significantly improve the overall functional level and daily living ability of patients. The rehabilitation effect of Taijiquan on paraplegic patients after spinal cord injury has been confirmed by scholars. Taijiquan training can improve the motor function, sensory function, mental health and overall health of patients. Taijiquan is a traditional and effective exercise rehabilitation method. There are several forms of Taijiquan used in spinal cord injury.

\subsection{Vertical: including two forms of ground and water.}

Taijiquan on the ground. Taijiquan rehabilitation is an active movement under the control of the brain. It contains the advantages of modern rehabilitation training. At the same time, it applies the three activities of Taijiquan to different rehabilitation periods of paraplegia patients, so as to make their attention, breathing and movement harmonious and natural. In training, the "intentional movement" precedes the "action" by visual adjustment, with the waist spine as the axis, stressing the spiral movement of the trunk, the proximal end to drive the distal end, the trunk to drive the limbs, using the normal muscle groups of the upper limbs and trunk to drive the paralyzed muscle groups of the lower limbs, is a holistic and harmonious rehabilitation training method. Studies have shown that Tai Chi rehabilitation is a very effective rehabilitation therapy, which can significantly improve the motor function, sensory function, and self-care ability of patients.

Taijiquan in water. The effects of Tai Chi on the balance function of incomplete spinal cord injury in Jinlong et al. In August 2017. Methods From March to December 2015, 30 patients with 
incomplete spinal cord injury were treated with shadowboxing in water for 30 minutes, 5 times a week for 5 weeks. Training parameters: water temperature $36 \mathrm{C}$, water depth to the level of xiphoid, unable to stand in the water patients need to wear a special buoyancy belt. Do not do any warm-up exercises before entering the water, train $30 \mathrm{~min}$ in water, 5 times a week. Firstly, the symmetrical movement of six legs supporting and center of gravity not moving was taught in a one-to-one way for one week, including turning clouds and rains, swinging the tail of carp, three ups and downs, three ups and downs, three openings and three closures, and crucifixion; the asymmetrical movement of three legs supporting and center of gravity not moving, such as white crane's bright wings, left looking right, curling humerus, etc. Six asymmetrical movements, such as bristle piercing, left and right palm piercing, knee-twisting, tail-twitching, single whip and cloud hand, and six one-legged supporting movements, such as ear piercing, turning and hammer handing, such as closing, looking back at the moon, left and right foot pedaling, golden cock independence, etc. After a total of 21 types of Tai Chi action society, patients were trained for 5 weeks under the guidance of therapists. Research shows that Tai Chi can improve the balance function of incomplete spinal cord injury patients.

\subsection{Seat type: "ten wheelchair" taijiquan.}

The influence of Taijiquan on the body. Jin Mei et al. put forward "ten-style" wheelchair Taijiquan. By discussing the development of "ten-style" wheelchair Taijiquan in the study of spinal cord injury and rehabilitation of disabled people in the United States, and drawing lessons from the content of traditional Taijiquan, combined with the physical characteristics of spinal cord injury and disabled people, it was founded for further rehabilitation. Its significance is that it can be very good. To provide a feasible, easy to operate, simple and easy physical therapy means, thus effectively improving the body's function, restoring and maintaining the original body function, increasing the body's ability, improving the existing state, to achieve the purpose of rehabilitation. In this sense, "ten-style" wheelchair Taijiquan provides a good means for spinal cord injury and disabled people, it is worth further promotion and demonstration.

Psychological influence of Shadowboxing. Patients with spinal cord injury are prone to produce a lot of psychological disorders, and the detection rate of psychological diseases is higher than the general population. Studies have shown that $74.4 \%$ of patients with spinal cord injury have anxiety and $77.9 \%$ have depression. The scores of somatization, compulsion, interpersonal relationship, depression, anxiety, hostility, phobia and psychiatric factors in patients with spinal cord injury paraplegia were higher than those in normal subjects, and the incidence of depression in patients with spinal cord injury was $94.9 \%$. Many patients with spinal cord injury have accepted the psychotherapy of Taijiquan and softball. Kim Mei et al. Observed the influence of "ten wheelchair" Taijiquan on mental health of patients with spinal cord injury. A 42-year-old male patient with spinal cord injury was treated with "ten-style" wheelchair Taijiquan for 90 minutes three times a week for 10 weeks. Results After 10 weeks, the factors of sleep, diet, somatization, hostility, paranoia, psychosis and obsessive-compulsive symptoms were significantly improved, and the factors of interpersonal sensitivity, depression, anxiety and phobia were also improved. The research shows that "ten-style" wheelchair Taijiquan has a good intervention effect on the mental health of patients, can effectively reduce or eliminate the breadth and depth of mental health problems, improve and improve the mental health of patients. Practicing "Ten-style" wheelchair shadowboxing requires mind-directed movements, and attention to breathing, mind and muscle relaxation. Through the spinal cord injury and the disabled sitting practice Taijiquan, can obtain comprehensive physical and psychological exercise, thus achieving efforts to improve the quality of life of spinal cord injury and disabled individuals.

\section{Taijiquan Improves the Mechanism of Paraplegia After Spinal Cord Injury}

After spinal cord injury, the ability of residual neurons to regenerate and replace neural connections is very limited. Therefore, important compensatory mechanisms, including maximizing the function of undamaged structures and pathways to compensate for lost tissues, are called neural remodeling, and are also the main recovery mechanisms after spinal cord injury. Neural remodeling is 
defined as "an adaptive reorganization of neural pathways that occurs after injury to partially restore lost function". This "recombination" has gradually been recognized as a major recovery mechanism after central nervous system injury (including spinal cord injury). The spinal cord is a low-grade part of the central nervous system, which is as plastic as the brain. Studies have shown that the plasticity changes after spinal cord injury have the same developmental and regional characteristics as the brain. If most of the dorsal roots of the cat's hind limbs were removed, the projection density of the well-preserved dorsal root nerve fibers in the spinal cord was increased, indicating a plasticity change between the reserved dorsal root and the adjacent resected dorsal root. Exercise is the most effective form of stimulation of the central nervous system. All movements provide sensory, motor and reflex inputs to the central nervous system. The spinal cord is an important pathway for the transmission of motor sensation between the brain and the body. Therefore, conditioned stimulation formed by repeated training is conducive to the occurrence of spinal plasticity, promote the regeneration of axonal protrusion buds, and thus improve the function of the spinal cord. Taijiquan, as a kind of dynamic exercise alternating muscle contraction and relaxation, improves the local blood supply of the affected limb by "moving all the time", and inputs a large amount of information to the central nervous system to promote the recovery and compensation of neuronal function, so that the function can be reconstructed to the greatest extent. Promote the remodeling of nervous system function, thus forming a new neural pathway, and then promote the functional recovery of patients with spinal cord injury.

\section{Taijiquan Applied To The Rehabilitation Of Hemiplegia After Spinal Cord Injurythe Application Of Taijiquan In The Rehabilitation Of Spinal Cord Injury Has a Good Theoretical Basis.}

At present, some clinical studies at home and abroad have initially confirmed that Taijiquan can improve the functional recovery of patients with spinal cord injury. However, there are still many deficiencies in the existing research. At present, there are only a few articles about the integration of Taijiquan into the rehabilitation training of spinal cord injury at home and abroad, but there are few articles about cervical spinal cord injury. The only action used in Taijiquan is the Cloud Hand Movement, which reflects the weak understanding of the movement of Taijiquan, so it can affect the therapeutic effect of Taijiquan in the process of spinal cord injury rehabilitation. Ten-style wheelchair Taijiquan perfects the training system of Taijiquan movement, but the proposition of ten-style wheelchair Taijiquan is mainly aimed at the patients in the wheelchair, and is not suitable for the patients with spinal cord injury who can walk independently in the late recovery period; and this part of the content, although the Taijiquan rehabilitation method has been involved, but the specific application of only the cloud hand. It reflects the incomplete understanding of Taijiquan Exercises in patients with spinal cord injury. In water taijiquan, the movement design considers the relationship between double leg support and single leg support, center of gravity movement and immobility, symmetry and asymmetry. The balance ability of the patients with incomplete spinal cord injury can be improved by adapting them to different conditions to practice, but the training in water needs certain conditions. It is not convenient to operate, so it is not convenient to popularize.

\section{Conclusions}

To sum up, because most of the researchers are from medical background, there is no deep insight into Taijiquan, only part of it can be used, not the whole method. Thus limiting the effect of Taijiquan on the recovery of cervical spinal cord injury. How to combine the complete training methods and theoretical system of Taijiquan with the characteristics of patients with spinal cord injury in different stages needs further research and exploration.

\section{References}

[1] Fact report. Spinal cord injury [EB/OL]. World Health Organization-Media Center 
http://www.who.int/mediacentre/factsheets/fs384/zh/

[2] Zhang Mengli, Zhou Tiantian, Liu Chaohui, et al. Progress in the application of Taijiquan in the rehabilitation of spinal cord injury [J].Rehabilitation theory and practice in China, 2016, 22 (11): 1301-1303.

[3] Li Pinmei.Taiji Rehabilitation Therapy for Paraplegia after Spinal Cord Injury [D]: [Master's Dissertation].Jilin: Jilin University, 2006.

[4] Jinlong, Congfang, Song Guiyun, et al. [J] Effect of Taiji in water on balance function in patients with incomplete spinal cord injury [J].Chinese Rehabilitation Theory and Practice, 2017, 23 (8): 955-958.5] Jinmei, Wang Yongtai, Marina, et al. [J] Development and preliminary application of "Ten-style" wheelchair Taijiquan in patients with spinal cord injury and disability [J]. Yu, 2011, 5 (21): 153 - 155.

[6] Jinmei, Marina, Qi Xiaoying, et al. [J] A case study on the psychological health of patients with spinal cord injury treated by "ten-style" wheelchair Taijiquan. Rehabilitation theory and practice in China, 2012, 418 (4): 386-388.

[7] Li Jianan, Xu Guangxu. Practical spinal cord injury rehabilitation [M]. Beijing: People's military medical press, 2013.

[8] Zhou Tianjian, Li Jianjun. Modern rehabilitation and treatment of spinal cord injury [M].Beijing: People's Health Publishing House, 2006.

[9] Li Jianjun, Yang Mingliang, Yang Degang, et al. Expert consensus on evaluation, treatment and rehabilitation of traumatic spinal cord injury [J]. Rehabilitation theory and practice in China 2017, 23 (3): 274-287.

[10] Shi Suhua, Li Zhigang, Song Jinling, et al. Effect of Electroacupuncture on the expression of cas-pase-3, calpastatin and myelin sheath changes after spinal cord injury in rats [J].Journal of Guangzhou University of Traditional Chinese Medicine, 2011, 28 (2): 149-154.

[11] Chen EW, Fu AS, Chan KM, et al. The efects of Tai Chi on the balance control of elderly persons with visual impairment: a randomised clinical trial [J]. Age and Age-ing, 2012, 41 (2): 254-259. 